\title{
Pleomorphic carcinoma of the lung: A surgical outcome
}

\author{
Tsuyoshi Yuki, MD, ${ }^{\text {a }}$ Toshiko Sakuma, MD, PhD, ${ }^{\text {b }}$ Chiho Ohbayashi, MD, PhD, ${ }^{\text {b,c }}$ Masahiro Yoshimura, MD, PhD, ${ }^{d}$ \\ Noriaki Tsubota, MD, PhD, ${ }^{a}$ Yutaka Okita, MD, PhD, ${ }^{d}$ and Morihito Okada, MD, PhD ${ }^{a}$
}

From the Departments of Thoracic Surgery $^{\mathrm{a}}$ and Pathology, ${ }^{\mathrm{b}}$ Hyogo Medical Center for Adults, Akashi City, Hyogo, Japan; and the Departments of Surgical Pathology, ${ }^{\mathrm{c}}$ and Cardiothoracic Surgery, ${ }^{\mathrm{d}}$ Kobe University Medical School, Kobe City, Hyogo, Japan.

Received for publication Feb 14, 2007; revisions received April 10, 2007; accepted for publication April 16, 2007.

Address for reprints: Morihito Okada, MD, PhD, Department of Surgical Oncology, Research Institute for Radiation Biology and Medicine, Hiroshima University, 1-2-3 Kasumi, Minami-ku, Hiroshima City, Hiroshima, 734-8553, Japan (E-mail: morihito1217jp@aol.com).

J Thorac Cardiovasc Surg 2007;134:399-404 $0022-5223 / \$ 32.00$

Copyright $\odot 2007$ by The American Association for Thoracic Surgery

doi:10.1016/j.jtcvs.2007.04.018
Objectives: Pleomorphic carcinoma of the lung, a rare malignant disease with a dual-cell component of spindle and/or giant cells, and of epithelial cells, was defined in the World Health Organization classification updated in 1999. Reported prognoses are heterogeneous, and optimal treatment remains undefined.

Methods: Data were retrospectively examined for 45 consecutive patients (41 men and 4 women) who had undergone surgical resection for pulmonary pleomorphic carcinoma.

Results: Sarcomatous elements were as follows: 23 spindle cell types (51.1\%), 11 giant cell types (24.4\%), and 11 combined spindle and giant cell types (24.4\%). Epithelial components were adenocarcinoma in $25(55.6 \%)$ patients, squamous cell carcinoma in $8(17.8 \%)$ patients, and large call carcinoma in $12(26.7 \%)$ patients. Nodal status was classified as pN0 disease in $28(62.2 \%)$ patients, pN1 disease in 5 $(11.1 \%)$ patients, and pN2 disease in $12(26.7 \%)$ patients. Even patients with pN0 disease frequently manifested vascular invasion (16/28 [57.1\%]). Five-year overall survival and disease-free survival were $39.2 \%$ and $47.1 \%$, respectively. Although the subtype of epithelial components, as well as sarcomatous elements, did not affect prognosis, overall survival $(P=.02)$ and disease-free survival $(P=.002)$ in patients with $\mathrm{pN} 1 / \mathrm{N} 2$ disease were significantly worse than those in patients with pN0 disease. Most recurrences occurred at distant sites (14/20 [70.0\%]), and recurrence within 6 months after resection was found in 10 patients (10/20 [50.0\%]). Moreover, median survival time after confirmation of initial relapse was 2.6 months.

Conclusions: Pulmonary pleomorphic carcinoma, which often presented in symptomatic male smokers as a large peripheral lesion, carried a poor prognosis, even when early-stage disease was diagnosed and resected. Distant metastases occurred more frequently and earlier, and the survival after relapse was very short, suggesting that this entity should be considered to have a tremendously aggressive malignant behavior. Further investigation of biologic features of pulmonary pleomorphic carcinoma and therapeutic response is a high-priority issue, so that suitable treatment strategies can be planned.

$\mathrm{T}$ he presence of sarcomatoid or pleomorphic elements with spindle or giant cells is an infrequent but well-recognized characteristic of some non-small cell lung cancers (NSCLCs). Before 1999, pathologists confronted with such tumors repeatedly experienced difficulties in providing a definite diagnosis, mainly because of the lack of standardization in terminology and diagnostic criteria. In the World Health Organization classifications revised in 1999, a group of poorly differentiated NSCLCs that have sarcomatous or sarcoma-like elements are described. Examples of pleomorphic carcinoma are NSCLC; squamous cell carcinoma; adenocarcinoma; large-cell carcinoma containing spindle cells, giant cells, or both; or a carcinoma consisting only of spindle cells and giant cells. ${ }^{1,2}$ The pleomorphic component should comprise at least $10 \%$ of the neoplasm. Giant cell carcinoma is restricted to a malignancy that is exclusively composed of giant cells, 


\section{Abbreviation and Acronym}

NSCLC $=$ non-small cell lung cancer

and spindle cell carcinoma is limited to that constituted of only spindle cells. Most fall into the category of pleomorphic carcinomas because of the presence of a double-cell component, and their pure forms are extremely uncommon. ${ }^{3}$

The actual nature of pleomorphic carcinoma has been debated extensively because of its rarity. In particular, its malignant grade and clinical behavior have not yet been defined. Two conflicting results are available on prognosis. Some investigations reported pleomorphic carcinoma to have a more aggressive clinical course and significantly poorer survival in comparison with other NSCLCs, ${ }^{4,5}$ but others failed to demonstrate any poorer prognosis for pleomorphic carcinomas. ${ }^{3,6}$ Several case reports but very few studies have been published regarding surgical resection of pulmonary pleomorphic carcinomas. However, to verify the proper selection of treatment strategy for this tumor, an understanding of its clinicopathologic behavior and prognosis is crucial. In view of the rarity and the significance of the histologic diagnosis, this study was accomplished in a retrospective, two-institutional setting with a critical review of pathology by an expert panel. We examined the clinicopathologic features of patients with pulmonary pleomorphic carcinoma and extensively analyzed surgical outcome.

\section{Materials and Methods}

We retrospectively examined data on 45 patients (41 men and 4 women) with lung pleomorphic carcinoma who underwent surgical resection between March 1985 and August 2006. Medical records provided information on age, sex, presenting symptom, smoking habits, stage, and operation. Bronchoscopy was performed preoperatively to establish the diagnosis of malignancy and to determine the extent of invasion and adequacy of the remaining airway. Preoperative evaluation included physical examination, chest roentgenography, and tumor markers. Furthermore, contrastenhanced computed tomographic scans of the chest, abdomen, and brain and bone scintigraphy were executed routinely. Staging was done based on the new International Staging System for Lung Cancer. $^{7}$

The standard surgical technique was used and accompanied by routine systematic dissection or sampling of the hilar and mediastinal nodes in every case. Intraoperative frozen examination was done to determine the extent of resection and to assess nodal status. After surgical intervention, the patients were examined in the outpatient clinic at 3-month intervals for 3 years and thereafter at 1 -year intervals in general. Two dedicated pulmonary pathologists examined all specimens histopathologically and classified them according to the criteria set by the World Health Organization. Sarcomatoid components were classified as follows: spindle cell type, giant cell type, and the combined spindle and giant cell type. Epithelial elements were described also. An institutional review
Table 1. Clinicosurgical characteristics $(n=45)$

\begin{tabular}{lc}
\hline Factor & \\
\hline Age (y) & $39-80$ \\
Range & 65.8 \\
Mean & \\
Sex & 41 \\
Male & 4 \\
Female & \\
Smoking status & $38(16 / 22)$ \\
Smoker (current/former) & 7 \\
Nonsmoker & \\
Size of tumor (mm) & $23-170$ \\
Range & 50.1 \\
Mean & \\
Procedure & $2(4.4 \%)$ \\
Pneumonectomy & $39(86.7 \%)$ \\
Lobectomy & 6 \\
$\quad+$ Bronchoplasty & 19 \\
$\quad+$ Extended resection & $2(4.4 \%)$ \\
Segmentecomy & $2(4.4 \%)$ \\
Wedge resection &
\end{tabular}

board approved this retrospective study, and written informed consent for the surgical intervention was obtained from each patient.

The duration of overall survival was defined as the interval between the day of the operation and the date of death by any cause or the last follow-up date. Disease-free survival was the interval from the date of resection to the date of proved detection of recurrence or metastases. Distributions of disease-free interval and overall survival were estimated by using the Kaplan-Meier method and compared by using the log-rank test.

\section{Results}

Clinicosurgical characteristics of the tumor are demonstrated in Table 1. Patient age ranged from 39 to 80 years (mean, 65.8 years), and the male/female ratio was 10.3:1. Thirty-eight $(84.4 \%)$ patients were smokers. The main clinical symptoms at presentation were chest pain $(n=6)$ and fever $(\mathrm{n}=6)$. Tumor size ranged between 23 and $170 \mathrm{~mm}$ (mean, $50.1 \mathrm{~mm}$ ). In most cases the tumor was located in the upper lobes ( $\mathrm{n}=33$ [73.3\%]; right, 20; left, 13). Except for 4 cases, all tumors were located in peripheral parenchyma $(\mathrm{n}=41$ [91.1\%]). Preoperative pathologic assessment indicated adenocarcinoma in 20 patients, squamous cell carcinoma in 8 patients, large cell carcinoma in 4 patients, and undefined non-small cell carcinoma in 13 patients, demonstrating that we failed to make the exact diagnosis before surgical intervention in all the patients. Neoadjuvant chemotherapy was administered to 1 patient, and chemoradiotherapy was administered to 1 patient, but downstaging of the disease was not achieved in either patient. Surgical procedures included 39 lobectomies, 2 peumonectomies, 2 segmentectomies, and 2 wedge resections. Among the 39 
Table 2. Stage and histopathology $(n=45)$

\begin{tabular}{lc}
\hline Factor & \\
\hline Clinical stage & $5(11.1 \%)$ \\
IA & $16(35.6 \%)$ \\
IB & $2(4.4 \%)$ \\
IIA & $14(31.1 \%)$ \\
IIB & $7(15.6 \%)$ \\
IIIA & $1(2.2 \%)$ \\
IIIB & 0 \\
IV & \\
Pathologic stage & $5(11.1 \%)$ \\
IA & $9(20.0 \%)$ \\
IB & 0 \\
IIA & $16(35.6 \%)$ \\
IIB & $10(22.2 \%)$ \\
IIIA & $3(6.7 \%)$ \\
IIIB & $2(4.4 \%)^{*}$ \\
IV & \\
Sarcomatous elements & $23(51.1 \%)$ \\
Spindle & $11(24.4 \%)$ \\
Giant & $11(24.4 \%)$ \\
Spindle and giant & \\
Epithelial components & $25(55.6 \%)$ \\
Adenocarcinoma & $8(17.8 \%)$ \\
Squamous cell carcinoma & $12(26.7 \%)$ \\
Large cell carcinoma & \\
\hline
\end{tabular}

*Pulmonary metastases were identified in the other lobes.

lobectomies, 6 bronchoplastic procedures were carried out, and 19 extended resections were required because of invasion of the adjacent tissue, including muscle and rib $(\mathrm{n}=$ 13), parietal pleura $(n=2)$, diaphragm $(n=2)$, and vagal nerve $(\mathrm{n}=2)$. Interlobar invasion was observed in 3 patients. Complete resection (R0) was achieved in 39 (86.7\%) patients, and a microscopic positive resection margin (R1) was observed in $6(13.3 \%)$ patients, although no patient had a macroscopic incomplete resection.

Sarcomatous elements and epithelial components are shown in Table 2. Pathologic stages were as follows: stage IA in 5 patients, stage IB in 9 patients, stage IIB in 16 patients, stage IIIA in 10 patients, stage IIIB in 3 patients, and stage IV in 2 patients. Nodal status was classified as pN0 in $28(62.2 \%)$ patients, pN1 in $5(11.1 \%)$ patients, and pN2 in $12(26.7 \%)$ patients (Table 3). Microscopic invasions into the blood vessels were observed in 29 patients

Table 3. Vascular invasion, lymphatic permeation, and nodal status

\begin{tabular}{lrc}
\hline Nodal status & Vascular invasion & Lymphatic permeation \\
\hline pN0 $(n=28)$ & $16(57.1 \%)$ & $4(14.3 \%)$ \\
pN1 $(n=5)$ & $2(40.0 \%)$ & $1(20.0 \%)$ \\
pN2 $(n=12)$ & $11(91.7 \%)$ & $10(83.3 \%)$ \\
\hline
\end{tabular}
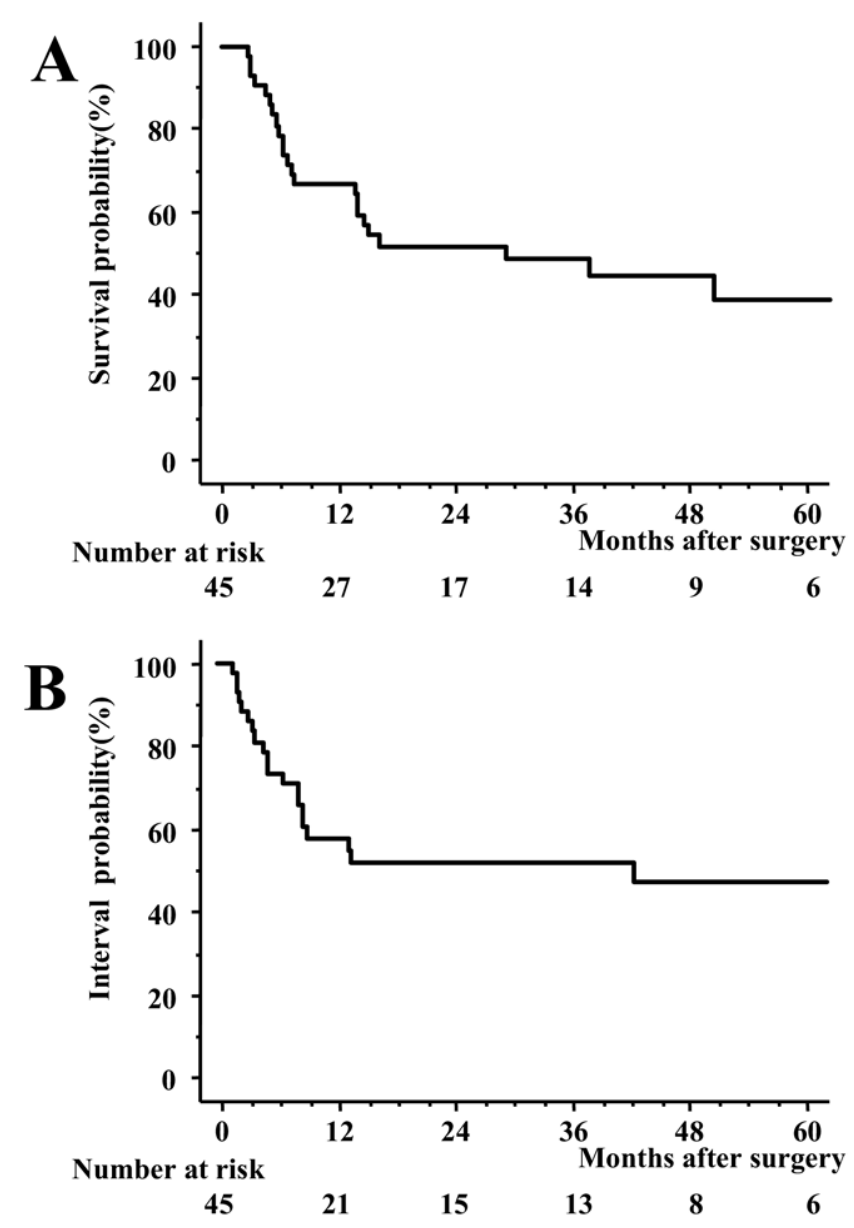

Figure 1. Overall survival curve $(A)$ and disease-free interval (B) for surgically resected pleomorphic carcinoma.

(29/45 [64.4\%]), and permeations of lymphatic vessels were found in 15 patients $(15 / 45$ [33.3\%]). Even patients with pN0 disease frequently had vascular invasion (16/28 [57.1\%]). Neither severe postoperative complications nor in-hospital mortality, including 30-day operative mortality, occurred in this series.

Median follow-up was 37 months. Three-year and 5-year overall survivals were $48.6 \%$ and $39.2 \%$, respectively (Figure $1, A$ ), whereas disease-free survival was $51.9 \%$ at 3 years and $47.1 \%$ at 5 years (Figure 1,B). There were statistically significant differences between the pN0 and $\mathrm{pN} 1 / \mathrm{N} 2$ groups in overall survival $(P=.02)$ and diseasefree interval $(P=.002$, Figure 2$)$. The subtype of epithelial components or sarcomatous elements did not affect overall survival or disease-free interval (Figures 3 and 4). Among 20 patients with recurrent disease, $14(70.0 \%)$ initially had distant metastasis, and 6 (30.0\%) had locoregional relapse (Table 4). The major sites of metastasis firstly identified were bone (6/14 [42.9\%]) and brain (4/14 [28.6\%]). All the 

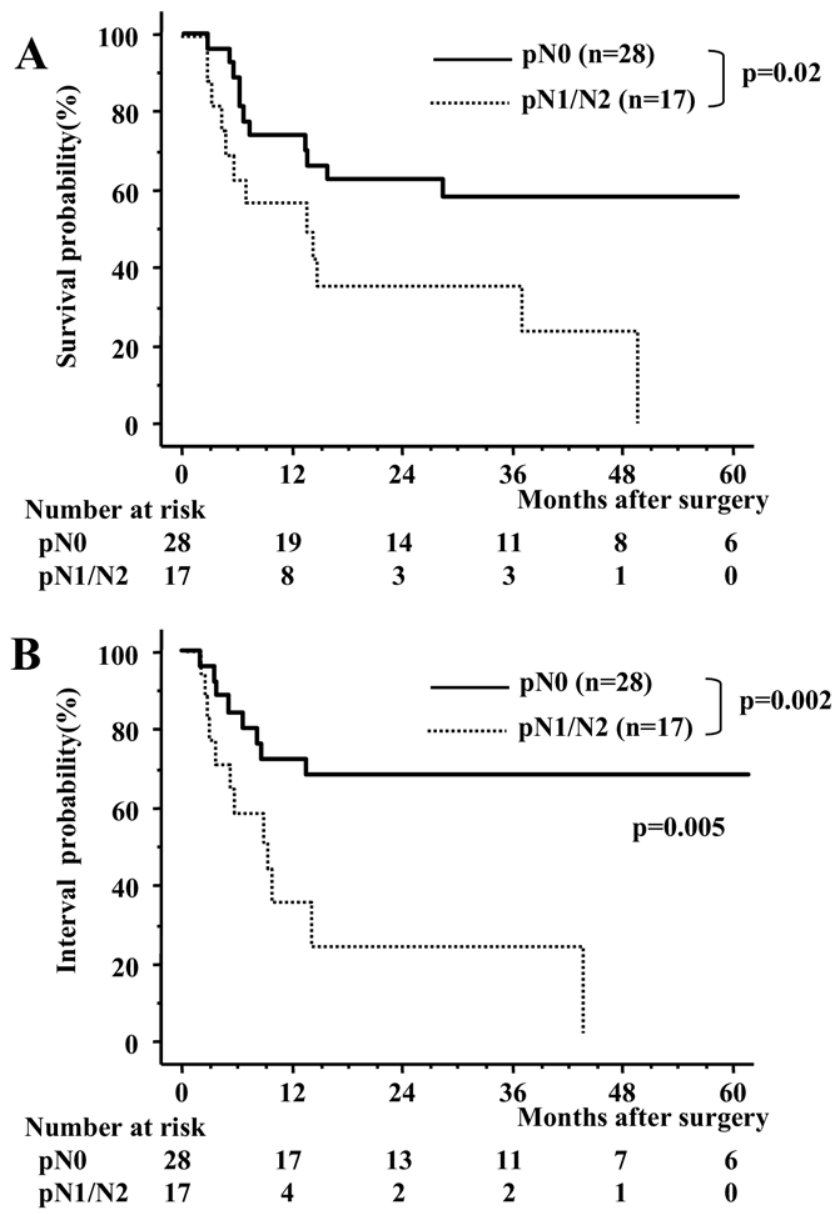

Figure 2. Overall survival curve (A) and disease-free interval (B) for surgically resected pleomorphic carcinoma according to pathologic nodal status.

distant metastases developed within 14 months after the operation, which included 8 patients with $\mathrm{pN} 0$ disease $(8 / 14$ [57.1\%]). Surprisingly, early postoperative relapse within 6 months was found in 10 patients $(10 / 20$ [50.0\%]), and furthermore, overall survival after confirmation of the initial relapse was $23.5 \%$ at 6 months, with a median survival time of 2.6 months.

\section{Discussion}

The true incidence of pulmonary pleomorphic carcinoma is, in all probability, low, although it has not been well defined. In our series $1.6 \%(45 / 2743)$ of resected NSCLCs were pleomorphic carcinomas. The majority of patients given diagnoses of pulmonary pleomorphic carcinoma were male and smokers who presented with symptoms, which is similar to patients in earlier studies. ${ }^{4,8,9}$ The pleomorphic carcinomas in this study, which ranged from 23 to $170 \mathrm{~mm}$, with an average greatest dimension of $50 \mathrm{~mm}$, tended to be
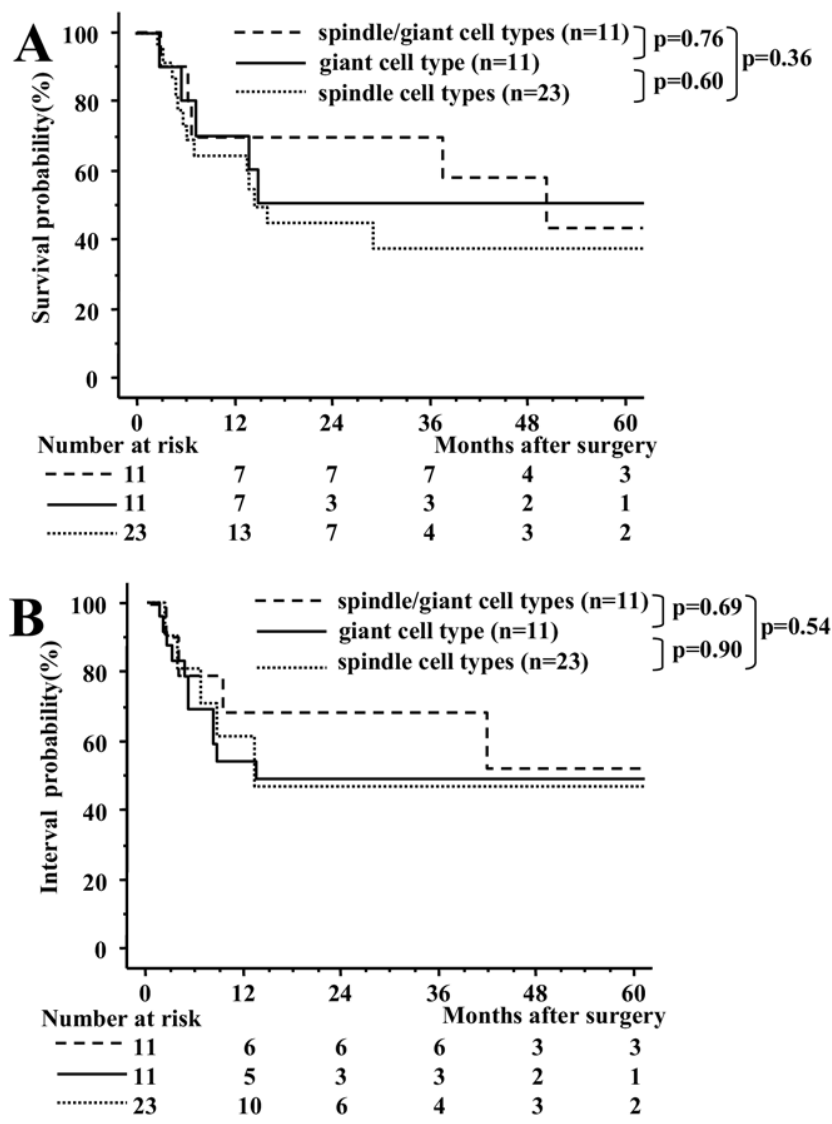

Figure 3. Overall survival curve (A) and disease-free interval (B) for surgically resected pleomorphic carcinoma according to sarcomatous elements.

large peripheral tumors. Because of a relatively large tumor potentially invading adjacent tissue, patients clinically presented with tumor-related symptoms, such as chest pain. Rossi and coworkers ${ }^{9}$ reported that cough, hemoptysis, or both were the most common presenting symptoms, occurring in $80 \%$ of patients, and that only $6.7 \%$ of patients had no symptoms at presentation. According to Fishback and colleagues, ${ }^{4} 65 \%$ of pleomorphic carcinomas were located in the upper lobes, $47 \%$ were in the right upper lobes, $60 \%$ were peripheral masses, and $24 \%$ had invaded the chest wall. Those findings were nearly consistent with those in our series. We note a predilection for the upper lobes, as in earlier series. ${ }^{10,11}$ Our data demonstrated that preoperative pathologic diagnosis in tiny tissues was complicated, which seemed to be caused by the heterogeneity and poor differentiation of the tumor cells. As a result, a definite diagnosis was finally established based on examination of surgically resected specimens. Also, the biphasic appearance of the tumor often makes diagnosis so difficult that immunohistochemical examination could be helpful in appropriate diagnosis. ${ }^{12}$ 


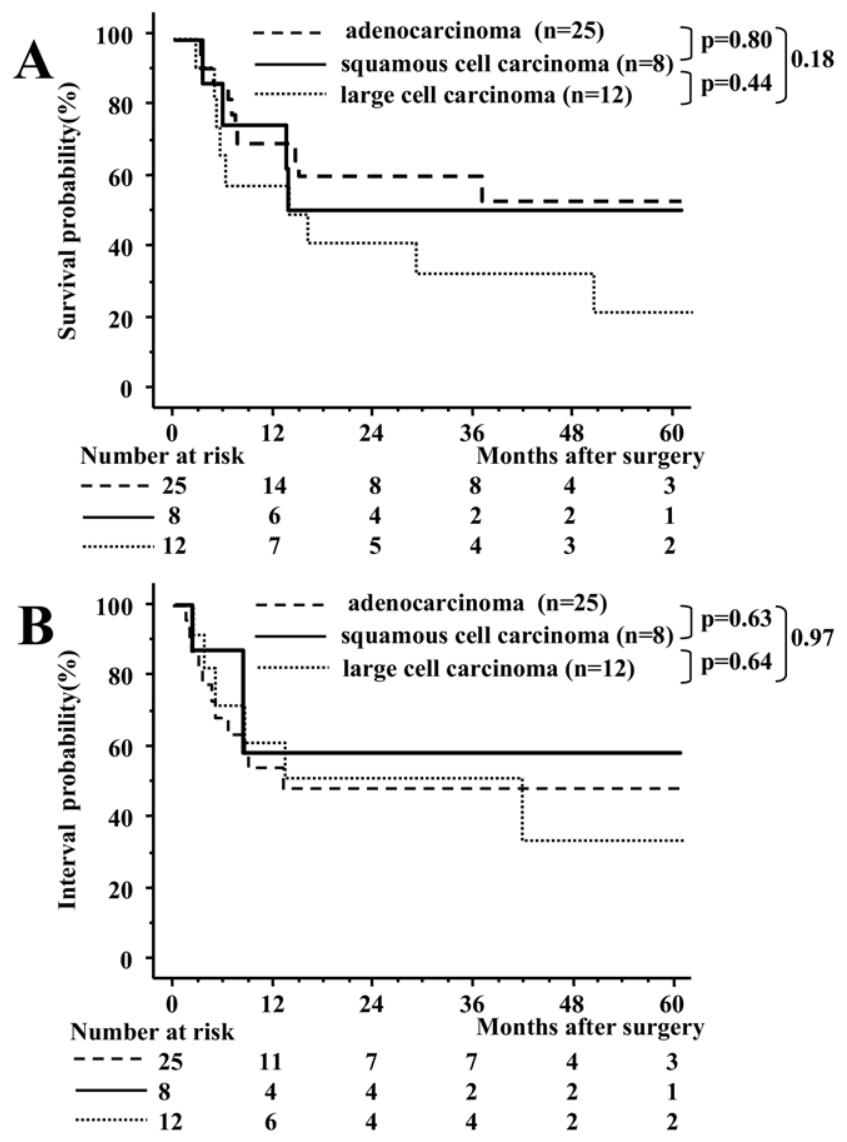

Figure 4. Overall survival curve (A) and disease-free interval (B) for surgically resected pleomorphic carcinoma according to epithelial components.

The clinical behavior and prognosis of pleomorphic carcinoma have seldom been discussed. Some reports showed a worse prognosis for patients with pleomorphic carcinoma compared with that of patients with other variants of NSCLCs, ${ }^{4,5}$ whereas Nakajima and associates ${ }^{6}$ reported similar clinical behaviors and prognosis between the 2 groups. $^{3}$ The most important implication of the present study is the determination of the malignant grade of this entity. The overall survival of patients with pathologic N0 pleomorphic carcinoma was $55 \%$ at 5 years, which was definitely worse than that of all patients with pathologic N0 NSCLCs ( $73 \%$ at 5 years). ${ }^{13}$ Also, the presence of pathologic nodal involvement negatively affected overall survival and disease-free interval, whereas interestingly, the subtype of epithelial components or sarcomatous elements did not influence prognosis. Patients with pN0 disease frequently had vascular invasion $(57.1 \%)$, indicating that distant metastases often occurred, even in early-stage disease. To our surprise, we found that almost half of all patients and one third of patients with pNO disease had died within 18
Table 4. Sites of initial recurrence

\begin{tabular}{ll}
\hline Locoregional $(\mathrm{n}=6)$ & \\
Mediastinal metastasis & $4(66.7 \%)$ \\
Pleural dissemination & $2(33.3 \%)$ \\
Distant $(\mathrm{n}=14)$ & \\
Bone & $6(42.9 \%)$ \\
Brain & $4(28.6 \%)$ \\
Lung & $2(14.3 \%)$ \\
Liver & $1(7.1 \%)$ \\
Adrenal gland & $1(7.1 \%)$ \\
\hline
\end{tabular}

months of the operation and that almost half of all patients and a third of patients with pN0 disease had a recurrence within 14 months. These disappointing data suggest that pulmonary pleomorphic carcinoma might be fatal, even at an early stage, unlike other NSCLCs. Notably, among 20 patients with relapse, including 8 patients with pN0 disease, 19 patients had recurrent disease within 14 months after the operation (95.0\%), and moreover, 6-month survival after initial proof of postoperative recurrence was a miserable $23.5 \%$. These results showed that pleomorphic carcinoma might be extremely aggressive in contrast with other NSCLCs.

The limitation of the present study should be mentioned: analysis in resectable cases to make sure that a methodical examination of pathologic characteristics would be possible. Therefore advanced unresectable lesions were inevitably excluded. Future investigations should incorporate advanced disease, even though it is difficult to obtain sufficient specimens by using biopsy samples and to make a definitive diagnosis in patients without surgical intervention. Results of this study showed difficulties in achieving a preoperative diagnosis. Pleomorphic carcinoma has been recognized as a form of NSCLC, and at present, the therapeutic strategy is the same as that for cases of NSCLC. Little information is available on systemic treatment options for this tumor entity, but basically, chemotherapy is considered ineffective because of the experienced poor sensibility of these types of tumors. Because of poor prognosis, combination of extensive surgical intervention resection with aggressive postoperative chemotherapy, radiotherapy, or both might be a reasonable consideration to improve survival. Further largescale assessment of the therapeutic response of pleomorphic carcinoma, including that to surgical intervention, chemotherapy, or radiation, is a high-priority issue that will determine the planning of appropriate treatment strategy.

\section{References}

1. Travis WD, Colby TV, Corrin B, Shimosato Y, Brambilla E, Sobin LH. World Health Organization International Histological Classification of Tumors. Histological typing of lung and pleural tumors. 3rd ed. Berlin: Springer-Verlag; 1999. 
2. Travis WD. Pathology of lung cancer. Clin Chest Med. 2002;23: 65-81.

3. Attanoos RL, Papagiannis A, Suttinont P, Goddard H, Papotti M, Gibbs AR. Pulmonary giant cell carcinoma: pathological entity or morphological phenotype? Histopathology. 1998;32:225-31.

4. Fishback NF, Travis WD, Moran CA, Guinee DG Jr, McCarthy WF, Koss MN. Pleomorphic (spindle/giant cell) carcinoma of the lung. A clinicopathologic correlation of 78 cases. Cancer. 1994; 73:2936-45

5. Raveglia F, Mezzetti M, Panigalli T, Fria S, Giuliani L, Conforti S, et al. Personal experience in surgical management of pulmonary pleomorphic carcinoma. Ann Thorac Surg. 2004;78:1742-7.

6. Nakajima M, Kasai T, Hashimoto H, Iwata Y, Manabe H. Sarcomatoid carcinoma of the lung: a clinicopathologic study of 37 cases. Cancer. 1999;86:608-16.

7. Mountain CF. Revisions in the International System for Staging Lung Cancer. Chest. 1997;111:1710-7.
8. Haque S. Carcinosarcoma of the lung: report of a case and review of the literature. J Pak Med Assoc. 1980;30:91-8.

9. Rossi G, Cavazza A, Sturm N, Migaldi M, Facciolongo N, Longo L, et al. Pulmonary carcinomas with pleomorphic, sarcomatoid or sarcomatous elements: a clinicopathologic and immunohistochemical study of 75 cases. Am J Surg Pathol. 2003;27:311-24.

10. Ginsberg S, Buzaid A, Stern H, Carter D. Giant cell carcinoma of the lung. Cancer. 1992;70:606-10.

11. Edwards CW. Pulmonary adenocarcinoma: review of 106 cases and proposed new classification. J Clin Pathol. 1987;40:125-35.

12. Chang YL, Lee YC, Shih JY, Wu CT. Pulmonary pleomorphic (spindle) cell carcinoma: peculiar clinicopathologic manifestations different from ordinary non small cell carcinoma. Lung Cancer. 2001;34:91-7.

13. Okada M, Nishio W, Sakamoto T, Uchino K, Yuki T, Nakagawa A, et al. Evolution of surgical outcomes for nonsmall cell lung cancer: Time trends in 1465 consecutive patients undergoing complete resection. Ann Thorac Surg. 2004;77:1926-31. 